\title{
Effects of Plasmin on von Willebrand Factor Multimers \\ Degradation In Vitro and Stimulation of Release In Vivo
}

\author{
Karen K. Hamilton, Larry J. Fretto, David S. Grierson, and Patrick A. McKee \\ The Howard Hughes Medical Institute Laboratories, Departments of Medicine and Biochemistry, \\ Duke University Medical Center, Durham, North Carolina 27710
}

\begin{abstract}
von Willebrand factor (vWF), a multimeric protein that mediates platelet adhesion, circulates in association with the procoagulant Factor VIII (FVIII). In previous reports, plasmin was shown in vitro to inactivate FVIII and cleave the vWF subunit extensively, but to cause only a modest decrease in vWF platelet-agglutinating activity. In the present study, the digestion of vWF multimers by plasmin was analyzed by sodium dodecyl sulfate-agarose gel electrophoresis and radioimmunoblotting. In vitro, plasmin degraded the large vWF multimers to smaller forms that could be distinguished from the small multimers present before digestion only by a slightly increased electrophoretic mobility. These plasmin-cleaved "multimers" were composed of disulfide-linked fragments with no intact $\mathrm{vWF}$ subunits. Thus, many plasmin cleavages occur within disulfide loops. The slight increase in mobility of plasmin-digested vWF is in part explained by the early cleavage from the multimers of a 34,000-mol wt peptide, which was purified and partially sequenced. The amino-terminal sequence (33 residues) agrees with the previously reported sequence (15 residues) for the amino terminus of the intact $v W F$ subunit. Analysis of plasmin-digested vWF allowed deduction of a model for the native vWF structure, including the approximate location of the interprotomer disulfide bond(s).

To determine whether plasmin would digest $\mathrm{vWF}$ in vivo, plasmas from 12 patients and 2 normal volunteers who received intravenous streptokinase (SK) were analyzed. Rather than vWF digestion, a two- to threefold rise in vWF antigen and platelet-agglutinating activity occurred within $2 \mathrm{~h}$ after a single SK dose, and the increase was greatest among the largest multimers. In contrast, FVIII clotting activity dropped to 10$20 \%$ of pre-SK levels. Thus, although plasmin destroys FVIII, a pharmacologically induced fibrinolytic state is associated with significant release of $\mathbf{v W F}$ from endothelial cells, platelets, or some other storage pool.
\end{abstract}

\section{Introduction}

Factor VIII/von Willebrand factor (FVIII/vWF) ${ }^{1}$ is a plasma protein complex with two hemostatic functions. Although

Dr. McKee is an Investigator of the Howard Hughes Medical Institute. Address reprint requests to Dr. McKee, Box 3705, Duke Hospital, Durham, NC 27710.

Received for publication 26 December 1984.

1. Abbreviations used in this paper: CTA, Committee on Thrombolytic Agents; FVIII, Factor VIII; FDP, fibrin-fibrinogen degradation products; PAGE, polyacrylamide gel electrophoresis; SK, streptokinase; vWF, von Willebrand factor.

J. Clin. Invest.

(C) The American Society for Clinical Investigation, Inc. $0021-9738 / 85 / 07 / 0261 / 10 \$ 1.00$

Volume 76 , July $1985,261-270$ present in only trace amounts in the complex, the FVIII protein possesses the procoagulant activity lacking in hemophilia A. vWF, which is deficient or defective in von Willebrand's disease, mediates platelet adhesion to subendothelium and platelet agglutination in the presence of ristocetin (1). vWF circulates as a series of large multimers that range in size from $\sim 500,000-800,000$ to $12-20$ million mol wt, as estimated from electrophoretic mobilities in sodium dodecyl sulfate (SDS)-agarose or agarose-acrylamide gels (2-4) and from volumes on electron micrographs (5). The smallest form of vWF seen in plasma (the protomer) appears to be a dimer of 200,000-280,000-mol wt subunits, whereas the larger forms are currently presumed to be oligomers of this dimer (3).

Plasmin is a plasma serine proteinase responsible for thrombolysis. Its circulating zymogen form, plasminogen, is converted to plasmin by urokinase, by the streptokinaseplasminogen activator complex, as well as by other circulating or tissue-associated plasminogen activators. In vivo, the primary plasmin substrates are fibrin and fibrinogen. However, plasmin will selectively cleave arginyl- $X$ and lysyl- $X$ peptide bonds in other proteins in vitro. Several laboratories have described the effects of plasmin on FVIII/vWF (6-9). In these early studies, when purified FVIII/vWF was incubated with plasmin, FVIII clotting activity was destroyed within 5-15 min; however, the vWF ristocetin-cofactor platelet-agglutinating activity was relatively resistant, with $\sim 70 \%$ of the activity remaining even after $24 \mathrm{~h}$ of digestion $(6,9)$. When plasmin-degraded FVIII/ vWF was examined by SDS-polyacrylamide gel electrophoresis (SDS-PAGE), after disulfide bond reduction, the vWF subunit was found to be cleaved into multiple fragments, ranging in molecular weight from 15,000 to 185,000 , depending on the duration of digestion. There was not good agreement among the studies about the number of fragments in the terminal digest or their molecular weights. When nonreduced plasmindigested FVIII/vWF was analyzed, the protein did not enter 4-5\% polyacrylamide gels to any significant extent (6-9), but by crossed immunoelectrophoresis, the mobility was increased (10). Because the multimeric structure of vWF was not recognized at the time of those studies and in light of current, though still limited, understanding of vWF structure, we analyzed the effect of plasmin in vitro on vWF multimers and found that the pattern of degradation allowed several deductions about the structure of native vWF. In addition, we undertook to determine whether vWF is significantly degraded by plasmin in vivo, and unexpectedly found that a dramatic increase in the plasma vWF level occurred during thrombolytic therapy.

\section{Methods}

Plasma preparation. Plasma was prepared from freshly drawn whole blood anticoagulated with citrate (one part $3.8 \%$ sodium citrate, nine parts whole blood) by centrifugation at room temperature at $2,500 \mathrm{~g}$ for $30 \mathrm{~min}$. The normal pooled plasma, which was used as the standard for our FVIII and vWF assays, was obtained from five apparently healthy male donors with no personal or family history of bleeding tendencies. 
Purification of $F V I I I / v W F$. FVIII/vWF was purified from lyophilized intermediate purity (11) concentrates (American Red Cross) by polyethylene glycol 4000 precipitation and $4 \%$ agarose gel (Biogel A-15m; Bio-Rad Laboratories, Richmond, CA) chromatography as previously reported (12). Protein concentration was determined by the method of Lowry et al. (13).

Purification of plasminogen. Glu-plasminogen was obtained by affinity chromatography of fresh-frozen plasma on lysine-agarose (14). Protein concentration was calculated using a molecular weight of 92,000 and an extinction coefficient $\left(E_{280 \mathrm{~nm}}^{1 \%}\right)$ of 17.0.

Plasmin degradation of purified FVIII/vWF. Plasminogen (15 $\mu \mathrm{l}$, $3.2 \mathrm{mg} / \mathrm{ml}, 20$ Committee on Thrombolytic Agents [CTA] U/mg) was activated using $10 \mu \mathrm{l}$ (10 CTA U) of urokinase (Sterling-Winthrop Pharmaceuticals, Rensselaer, NY). After 15 min at room temperature, $1 \mathrm{ml}$ of purified $\mathrm{FVIII} / \mathrm{vWF}(2 \mathrm{mg} / \mathrm{ml})$ was added; digestion proceeded at $37^{\circ} \mathrm{C}$ and aliquots were taken from $5 \mathrm{~min}$ to $60 \mathrm{~h}$. Plasmin in each aliquot was inactivated by the addition of a 10-fold molar excess of aprotonin (Trasylol; FBA Pharmaceuticals, New York, NY). Controls containing FVIII/vWF plus buffer or urokinase (without plasminogen) were incubated simultaneously.

Trypsin degradation of purified FVIII/vWF. Trypsin- $N$-tosyl-Lphenylalanine chloromethyl ketone $(231 \mathrm{U} / \mathrm{mg}$; Millipore Corp., Bedford, MA) was dissolved in $1 \mathrm{mM} \mathrm{HCl}$, left at room temperature for $1 \mathrm{~h}$, and added to purified FVIII/vWF $(2 \mathrm{mg} / \mathrm{ml})$ at a final concentration of $8 \mu \mathrm{g} / \mathrm{ml}$. As was done for the plasmin degradation, aliquots were removed at intervals from $5 \mathrm{~min}$ to $60 \mathrm{~h}$ of digestion and trypsin inactivated with aprotonin in a 10-fold molar excess; a control (containing FVIII/vWF plus buffer) was incubated and sampled in parallel.

Isolation and characterization of the 34,000- mol wt plasmic peptide from $v W F$. vWF without FVIII activity was obtained from FVIII/vWF as previously described (15). Purified FVIII/vWF was mixed with oneninth part $2.5 \mathrm{M} \mathrm{CaCl}_{2}-0.02 \mathrm{M}$ Tris, $\mathrm{pH} 6.8$, and rechromatographed on $4 \%$ agarose columns $(2.6 \times 90 \mathrm{~cm})$ that had been equilibrated with $0.01 \mathrm{M}$ L-histidine- $0.25 \mathrm{M} \mathrm{CaCl}_{2}-0.10 \mathrm{M} \mathrm{NaCl}-0.001 \mathrm{M}$ benzamidine$\mathrm{HCl}, \mathrm{pH}$ 6.0. The FVIII-free vWF in the void volume was pooled precipitated with $12 \%$ polyethylene glycol 4000 , and redissolved at 2.5 $\mathrm{mg} / \mathrm{ml}$ in $0.05 \mathrm{M}$ Tris- $0.15 \mathrm{M} \mathrm{NaCl}, \mathrm{pH}$ 7.35. Plasmin at 0.74 CTA $\mathrm{U} / \mathrm{ml}$ (1.6\% of vWF by weight) was incubated with $200 \mathrm{mg}$ of vWF at $37^{\circ} \mathrm{C}$ with stirring for $4 \mathrm{~h}$, after which aprotonin was added to give 100 kallikrein inhibitor units/ml final concentration. The final incubation mixture $(81 \mathrm{ml})$ was then lyophilized, dissolved in $30 \mathrm{ml}$ of 4.0 M guanidine- $\mathrm{HCl}$, concentrated to $12 \mathrm{ml}$ (model YM30; Amicon Corp., Danvers, MA) and gel-filtered on Biogel A-15m $(2.6 \times 87 \mathrm{~cm})$ in $4 \mathrm{M}$ guanidine-0.05 $\mathrm{M}$ Tris- $0.15 \mathrm{M} \mathrm{NaCl}, \mathrm{pH}$ 7.35. Subsequent rechromatography was performed on Sephacryl S-200 (Pharmacia Fine Chemicals, Piscataway, NJ) in the same buffer. The final pool was exhaustively dialyzed against $0.1 \mathrm{M}$ acetic acid and lyophilized.

Amino acid analyses for 22- and 72-h hydrolysates $(6 \mathrm{~N} \mathrm{HCl}, 0.1 \%$ phenol; $110^{\circ} \mathrm{C}$ ) were performed as previously detailed (16). Aminoterminal sequence analysis was done on a gas-liquid solid-phase peptide sequenator (17)

Effect of plasmin on $v W F$ in plasma and clots in vitro. Plasma was clotted in glass tubes by recalcification with 0.05 volume of $0.5 \mathrm{M}$ $\mathrm{CaCl}_{2}$. After $30 \mathrm{~min}$ at $37^{\circ} \mathrm{C}, 0.05$ vol of buffer or streptokinase (SK) (Kabikinase, KabiVitrum, Stockholm, Sweden) was added to the clot. Final SK concentrations were $50,100,200,500$, and $2,500 \mathrm{U} / \mathrm{ml}$ plasma. Buffer-treated clots showed no evidence of lysis after $12 \mathrm{~h}$, but all SK-treated clots lysed within $1 \mathrm{~h}$. Similar experiments were performed in which SK or buffer was added to unclotted plasma, serum, or platelet-rich plasma. In some experiments, additional SK was added hourly for $6 \mathrm{~h}$. In one experiment, plasmin was activated in plasma by the addition of urokinase (final concentration 2,500 CTA U/ml plasma) rather than SK. Gel analyses of the vWF multimers in the treated and control samples were performed as described below.

Effect of "euglobulin" lysis on vWF. "Euglobulin" precipitates of plasma were prepared by diluting $0.5 \mathrm{ml}$ of plasma with $8 \mathrm{ml}$ of deionized $\mathrm{H}_{2} \mathrm{O}$ and adding $0.15 \mathrm{ml}$ of $1 \%$ acetic acid (final $\mathrm{pH} 5.6$ ), incubating at $4^{\circ} \mathrm{C}$ for $30 \mathrm{~min}$, centrifuging at $630 \mathrm{~g}$ for $5 \mathrm{~min}$ at $4^{\circ} \mathrm{C}$, and resuspending in $0.5 \mathrm{ml}$ of borate buffer $(1 \mathrm{~g}$ of $\mathrm{NaCl}, 9 \mathrm{~g}$ of $\mathrm{Na}$ borate in 1 liter $\mathrm{H}_{2} \mathrm{O}$, pH 9.0). Samples were either clotted by recalcification or mixed with aprotonin (control sample) and incubated at $37^{\circ} \mathrm{C}$ until the clotted sample lysed and then for an additional $4 \mathrm{~h}$. The vWF multimer pattern in the lysed sample was compared to that in the proteinase-inhibited control euglobulin fraction.

Evaluation of SK therapy on FVIII/vWF in vivo. Informed consent was obtained from 12 patients and two normal volunteers who were participating in a study of the pharmacokinetics of SK (Grierson, D. S., and T. D. Bjornsson, manuscript in preparation). Plasmas were obtained immediately before the initial dose of SK, during this dose at 5-15-min intervals, and every $30 \mathrm{~min}$ for the first $2 \mathrm{~h}$. Five patients with acute myocardial infarction received a single dose of 500,000 U of intravenous SK over $30 \mathrm{~min}$; FVIII clotting activity was not assessed in these patients because they received heparin during cardiac catheterization. Seven patients with deep vein thrombosis, pulmonary embolus, or superior vena cava occlusion received $250,000 \mathrm{U}$ of SK over $30 \mathrm{~min} ; 2 \mathrm{~h}$ later five of these patients were started on a continuous SK infusion at $100,000 \mathrm{U} / \mathrm{h}$ for $1-3 \mathrm{~d}$. The length of infusion was determined by the patient's diagnosis and the occurrence of complications. Two normal volunteers received $100,000 \mathrm{U}$ of SK over $15 \mathrm{~min}$. All subjects, except one of the normal volunteers, achieved a fibrinolytic state as manifested by decreased fibrinogen and plasminogen levels. No patient had evidence of a febrile or allergic reaction. vWF antigen and FVIII/vWF activity levels were measured (as described below) and compared with pretreatment levels. To be more certain that changes in FVIII/vWF were indeed due to SK therapy and were not due simply to stress or the effects of contrast injection, similar analyses were performed on plasmas from three patients undergoing cardiac catheterization and from four normal volunteers who received an intravenous dose of heparin $(5,000 \mathrm{U}$ over $10 \mathrm{~min}$ ).

Ristocetin cofactor activity. vWF-dependent platelet agglutination in the presence of ristocetin $(\mathrm{H}$. Lundbeck \& Co., Copenhagen, Denmark) was measured using paraformaldehyde-fixed platelets (18) as previously described (5). Final ristocetin concentration was 1.5 $\mathrm{mg} / \mathrm{ml}$.

FVIII procoagulant activity. FVIII procoagulant activity was measured using both the one-stage partial thromboplastin time (19) and the two-stage thromboplastin generation methods $(20,21)$. Thrombofax (Ortho Diagnostic Systems Inc., Raritan, NJ) was used as the phospholipid source. In addition, thrombin clotting times (21) were performed on patient plasmas to determine whether fibrin-fibrinogen degradation products (FDP) were present in concentrations sufficient to inhibit fibrin polymerization. To determine whether FDP might also be interfering with the FVIII assays, thrombin clotting times were performed on: $(a)$ the one-stage assay mixture containing diluted patient plasma, hemophilic substrate plasma, and Thrombofax; and (b) the two-stage assay mixture of diluted adsorbed patient plasma, normal serum, bovine Factor V, Thrombofax, and normal substrate plasma. All FVIII and vWF assay results were compared with normal pooled plasma, which was defined as containing $1 \mathrm{U}$ of each activity per milliliter.

Gel analysis of $v W F$. vWF multimers were analyzed by SDS-1.5\% agarose gel electrophoresis in Tris-borate buffer (12.1 g of Tris base, $3.7 \mathrm{~g}$ of boric acid per liter, $\mathrm{pH}$ 8.6). Proteins were transferred electrophoretically from the gels to nitrocellulose paper, reacted with affinity-purified ${ }^{125}$ I-labeled rabbit antibody to human vWF, and autoradiographed (Hamilton, K. K., J. G. Keck, L. J. Fretto, M. E. P. Switzer, and P. A. McKee, manuscript in preparation). Total vWF antigen and VWF antigen in various regions of the multimer distribution were quantitated by counting radioactivity bound to nitrocellulose in each sample lane or subregion of the sample lane. Samples were compared with normal pooled plasma which was defined as having $1 \mathrm{U}$ of vWF antigen per milliliter. Serial dilutions of this pooled plasma were included on each gel.

Samples of purified vWF taken before and during plasmin digestion were reduced with $5 \% \beta$-mercaptoethanol and analyzed by SDS-PAGE 


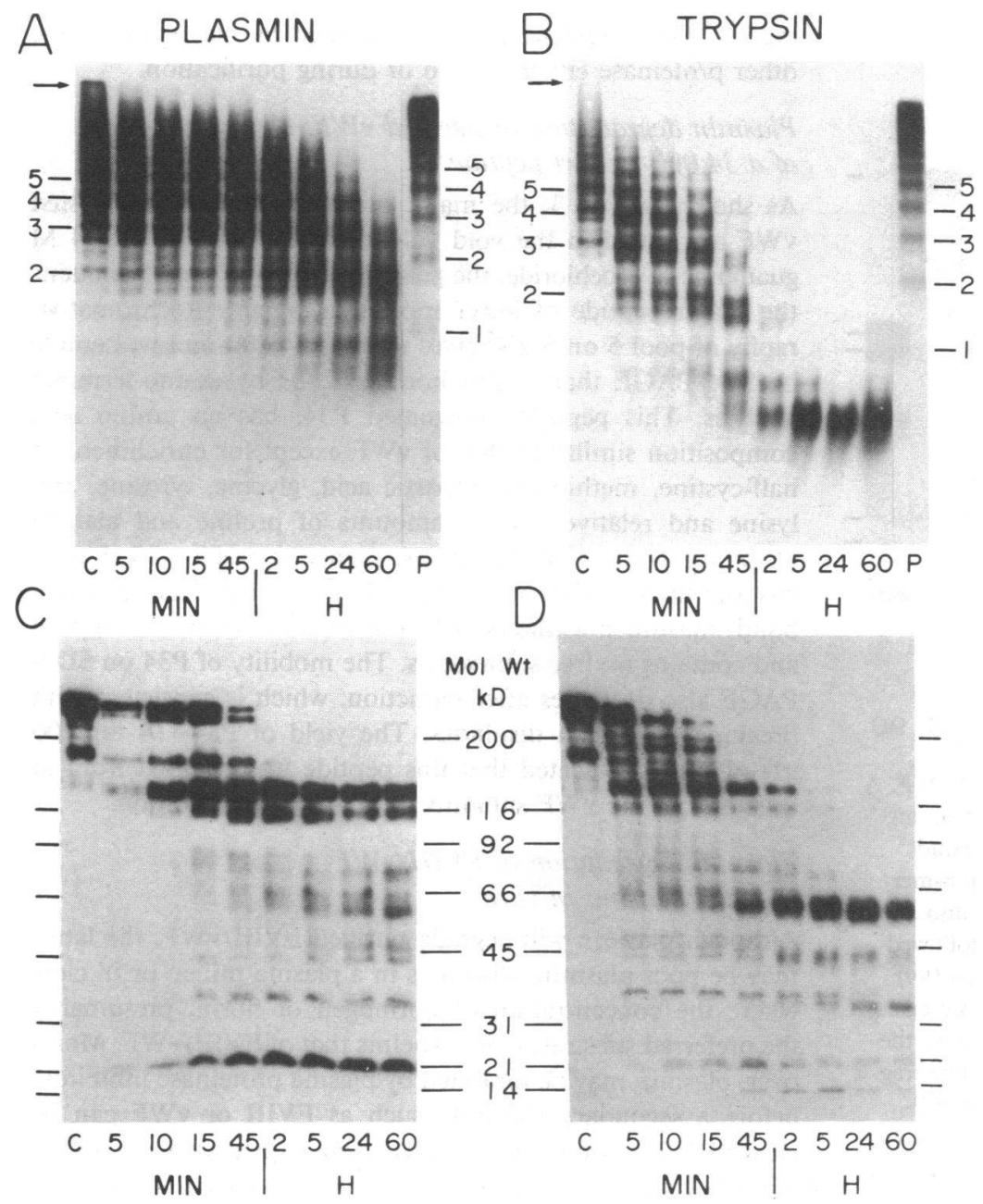

Figure 1. Plasmin- and trypsin-degraded vWF. $(A)$ Plasmin-degraded vWF analyzed (nonreduced) by SDS- $1.5 \%$ agarose gel electrophoresis and radioimmunoblotting. vWF was incubated with the proteinase for the times indicated. Plasma $(\mathrm{P})$ and control vWF incubated without enzyme $(C)$ were included on the same gel; multimers consisting of one to five protomers are indicated. The $34,000 \mathrm{~mol} w \mathrm{wt}$ degradation product is not seen on agarose gels. The gel origin is marked with a small arrow. (B) Trypsin-degraded vWF analyzed as described for $A$. $(C)$ Plasmin-degraded vWF analyzed after disulfide bond reduction by SDS-PAGE (5-12\% gradient) followed by radioimmunoblotting. Molecular weight standards are shown. A fragment of mol wt 168,000 , seen by immunoblotting in all our starting material (lane $C$ ), is also degraded by plasmin. $(D)$ Trypsin-degraded vWF (reduced) analyzed as in $C$.
(5-12\% gradient resolving gels with a $3.3 \%$ stacking gel), using the buffer system of Laemmli and Favre (22). These gels were either stained with Coomassie Blue or in selected instances with silver (23), or transferred to nitrocellulose, reacted with ${ }^{125}$ I-labeled rabbit antivWF, and autoradiographed.

Statistical analysis. To assess the effects of SK on vWF antigen, vWF ristocetin-cofactor activity, and FVIII clotting activity, all assay results were expressed as a percentage of the respective patient's pretreatment values. The median and the $95 \%$ confidence intervals for these medians at various times during the course of SK therapy were computed by using distribution-free estimators based on the Wilcoxon signed rank statistic (24).

\section{Results}

\section{Plasmin degradation of purified FVIII/vWF}

Effect on $v W F$ multimers. The time course of plasmin digestion of the purified vWF multimers, analyzed nonreduced by SDS$1.5 \%$ agarose gel electrophoresis and radioimmunoblotting, is shown in Fig $1 A$. The most striking feature was the progressive loss of the larger multimers and concordant increase in smaller forms. There was also a loss of sharpness in the original triplet pattern which was prominent in our purified vWF. The plasmin-degraded multimers migrated slightly faster than the corresponding multimers in the control sample, which can be explained by the loss of a small peptide $(34,000 \mathrm{~mol} \mathrm{wt})$ that was not seen on the large-pore agarose gels, but which was detected by SDS-PAGE of nonreduced samples. This fragment was much more prominent on Coomassie staining than on immunoblots, suggesting that it contains few binding sites for our rabbit antibody to native vWF.

Trypsin also degraded vWF from larger to smaller multimers (Fig. $1 B$ ), but much more rapidly than plasmin at the same molar concentration, which is consistent with the broader specificity of trypsin. Even with prolonged digestion and addition of more proteinase, vWF with fewer than three multimers was never produced by plasmin. In contrast, the terminal trypsin digest consisted of a major form that is smaller than the vWF protomer and many smaller fragments not visualized on agarose gels. The amount of radiolabeled antibody bound to each lane of the immunoblot shown in Fig. $1 \mathrm{~A}$ decreased during plasmin digestion, indicating gradual loss of antigenic sites ( $17 \%$ loss by $15 \mathrm{~min}, 31 \%$ by $5 \mathrm{~h}$ ). In previous reports, when "FVIII-related" antigen (i.e., vWF antigen) was measured by rocket immunoelectrophoresis, the rocket height after plasmin digestion increased $200-500 \%(6,7)$. Rocket immunoelectrophoresis may measure primarily the concentration of the fastest migrating form of vWF, which does increase dramatically as digestion proceeds (Fig. $1 \mathrm{~A}$ ). The greater loss of antigenicity with trypsin (25\% loss by $15 \mathrm{~min}, 82 \%$ by $5 \mathrm{~h}$ ) suggests more rapid degradation of native structure than occurs with plasmin. 


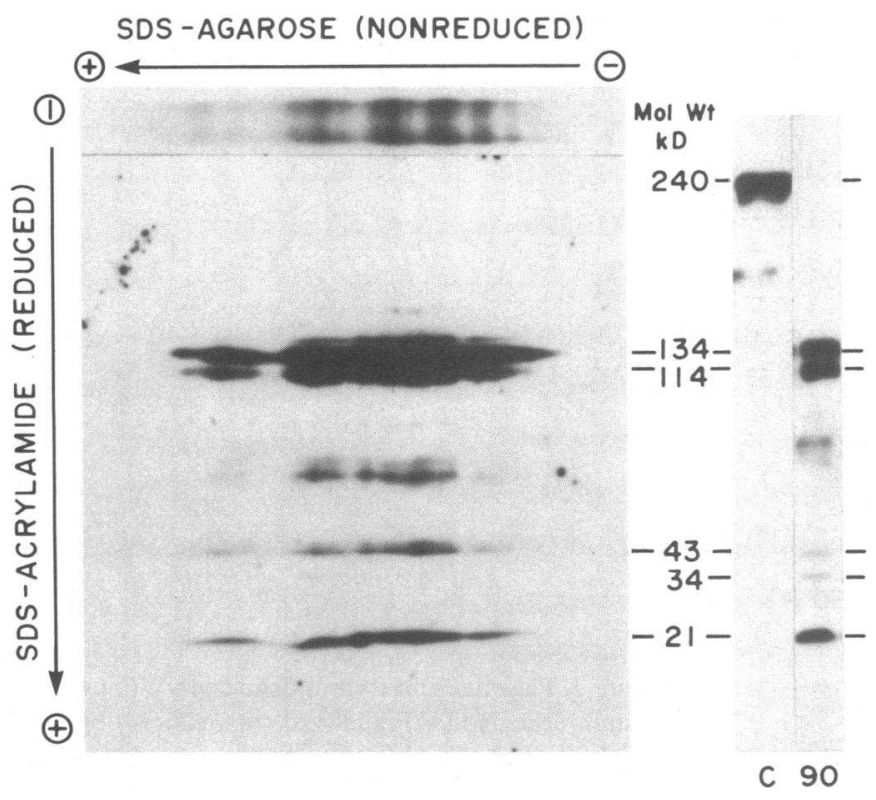

Figure 2. Two-dimensional gel analysis of plasmin-degraded vWF. A 90-min plasmin digest was first electrophoresed nonreduced on an SDS-1.5\% agarose gel (top horizontal lane). After disulfide bond reduction by incubation in Laemmli sample buffer with $5 \% \beta$-mercaptoethanol, the excised agarose lane was electrophoresed into an SDS-polyacrylamide gel with three layers $(5,8$, and $12 \%)$, followed by immunoblotting. The control vWF $(C)$ and 90 -min digest (90) were both analyzed after reduction on a similar gel in one dimension only for comparison (right). The nonreduced multimers seen in the 90-min digest (top) were composed predominantly of two large fragments $(134,000$ and $114,000 \mathrm{~mol} \mathrm{wt})$ with some $66,000-, 60,000-$, $48,000-, 43,000-$, and 21,000-mol wt fragments. The 34,000-mol wt fragment seen in the one-dimensional analysis of the reduced 90-min digest (right) is not present in the second dimension of the twodimensional gel, confirming that it is not disulfide-linked into the multimers. The 240,000-mol wt subunit seen in reduced control vWF $(C)$ is also not present in the multimers of the 90 -min digest.

Pattern of $v W F$ subunit cleavage. Plasmin- and trypsindegraded purified FVIII/vWF were also analyzed by SDSPAGE after disulfide bond reduction (Fig. $1 C$ and $D$ ). The major early plasmin digestion products were $134,000,114,000$, and $34,000 \mathrm{~mol} \mathrm{wt}$, with minor bands at 220,000, 168,000, 43,000 , and $21,000 \mathrm{~mol}$ wt (Fig. $1 \mathrm{C}$ ). With further digestion, all forms larger than $134,000 \mathrm{~mol} w t$ disappeared and the concentration of the 134,000 - and $114,000-\mathrm{mol}$ wt bands decreased as the intensity of the bands at 78,000, 66,000, $60,000,48,000,43,000$, and $21,000 \mathrm{~mol} \mathrm{wt}$ increased. Comparison of the nonreduced and reduced patterns (Fig. $1 A$ and $C$ ) suggests that many of the plasmin cleavage sites lie within disulfide loops, in that the large multimers persisted long after the intact subunit $(240,000 \mathrm{~mol} \mathrm{wt})$ had disappeared. Twodimensional SDS gel analysis of a 90-min digest (Fig. 2) shows conclusively that the plasmin-degraded vWF multimers are composed of multiple disulfide-linked fragments, rather than 240,000-mol wt subunits. The loss of the sharp triplet pattern seen on nonreduced gels (Fig. $1 A$ ) is therefore not surprising.

Of note, all of our purified FVIII/vWF preparations, even before plasmin treatment, contained variable amounts of a 168,000 -mol wt band which is easily seen on immunoblots (Fig. $1 C$, lane 1 ) and which intensified early in some plasmin digests. This fragment may be generated by plasmin or some other proteinase either in vivo or during purification.

\section{Plasmin degradation of purified $v W F$ : characterization of a 34,000-mol wt peptide}

As shown in Fig. 3, the major products of plasmin-digested vWF are eluted in the void volume of $4 \%$ agarose with $4 \mathrm{M}$ guanidine hydrochloride; the peak designated as pool 5 contains the major peptide of lower molecular weight. Rechromatography of pool 5 on S-200 gives a single $34,000-\mathrm{mol}$ wt peptide by SDS-PAGE that is also homogeneous by amino-terminal analysis. This peptide, designated P34, has an amino acid composition similar to that of vWF except for enrichment in half-cystine, methionine, aspartic acid, glycine, tyrosine, and lysine and relatively lesser amounts of proline and alanine (Table I). The amino-terminal sequence to residue 33 (Fig. 4) has four half-cystines which are involved in intrachain disulfide bonds inasmuch as the peptide was isolated without reduction and contains no free sulfhydryls. The mobility of P34 on SDSPAGE also decreases after reduction, which is consistent with breaking intrachain disulfides. The yield of P34 (14 mg/200 $\mathrm{mg}$ of vWF) indicated that this peptide was released from at least half of the vWF subunits during the $4 \mathrm{~h}$ digestion.

\section{Plasmin degradation of $F V I I I / v W F$ in whole plasma in vitro}

Although plasmin will degrade purified FVIII/vWF, the latter may be poor plasmin substrates in a plasma milieu or in clots where the concentration of fibrinogen or fibrin, presumably the preferred substrates, overwhelms that of FVIII/vWF. Moreover, plasmin may be inhibited by plasma proteinase inhibitors before a secondary substrate such as FVIII or vWF can be cleaved. When whole plasma or plasma clots were treated with selected concentrations of either SK or urokinase for 1-12 h, no change in the vWF multimer pattern was discerned. That active plasmin was in fact generated was verified by the rapid lysis of the fibrin clots. Moreover, FVIII clotting activity of the plasma was destroyed (only $15 \%$ remained 15 min after addition of $100 \mathrm{U}$ of SK per milliliter). To determine whether

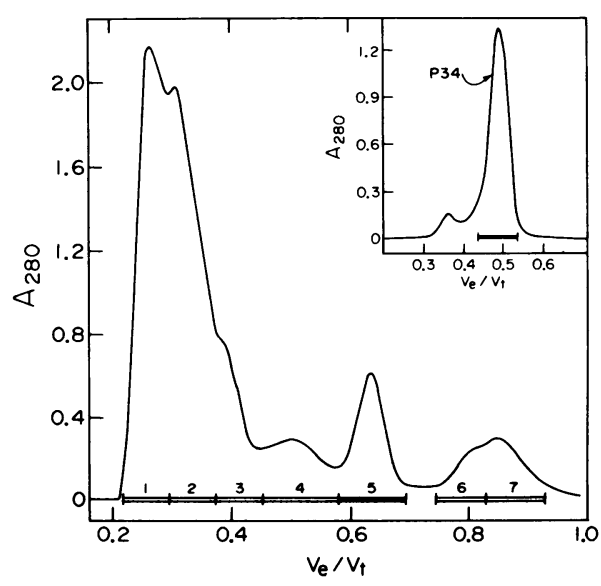

Figure 3. Purification of $\mathrm{P} 34$ from plasmin-digested vWF. The elution profile of whole 4-h digest on $4 \%$ agarose in $4 \mathrm{M}$ guanidine- $\mathrm{HCl}$, pH 7.0; pool 5 contains P34 (34,000-mol wt fragment). (Inset) Rechromatography of pool 5 on Sephacryl S-200 in $4 \mathrm{M}$ guanidine$\mathrm{HCl}, \mathrm{pH}$ 7.0. $A_{280}$, absorbance at $280 \mathrm{~nm} ; V_{\mathrm{e}}$, elution volume; $V_{\mathrm{t}}$, total column volume. 
Table I. Amino Acid Compositions*

\begin{tabular}{lrrr}
\hline Amino acid & $\begin{array}{l}\text { P34 } \\
\text { residues }\end{array}$ & P34 & vWF \\
\hline & \multicolumn{1}{l}{$n$} & mol\% & mol\% \\
Asp & 32.2 & 10.7 & 9.2 \\
Thr & 15.9 & 5.3 & 5.7 \\
Ser & 20.2 & 6.7 & 7.0 \\
Glu & 32.5 & 10.8 & 11.6 \\
Pro & 14.0 & 4.7 & 6.6 \\
Gly & 24.5 & 8.1 & 7.0 \\
Ala & 10.0 & 3.3 & 5.3 \\
1/2Cys & 23.8 & 7.9 & 7.0 \\
Val & 26.2 & 8.7 & 8.9 \\
Met & 5.4 & 1.8 & 1.4 \\
Ile & 9.9 & 3.3 & 3.7 \\
Leu & 24.0 & 8.0 & 7.6 \\
Tyr & 9.2 & 3.0 & 2.6 \\
Phe & 8.6 & 2.8 & 2.7 \\
His & 7.1 & 2.3 & 2.5 \\
Lys & 17.9 & 5.9 & 4.5 \\
Arg & 15.0 & 5.0 & 4.7 \\
Trp & 5.4 & 1.7 & 2.0 \\
& & & \\
\hline
\end{tabular}

* Amino acids were determined as described in Methods. Calculation of number of residues is based upon estimate of $34,000 \mathrm{~mol} w \mathrm{wt}$ from SDS-PAGE.

vWF might be digested when the fibrinolytic system is activated by naturally occurring circulating plasminogen activators, "euglobulin" precipitates were prepared just as for measuring euglobulin lysis times. This technique, which is based on precipitation of proteins at low ionic strength near their isoelectric points, yields fibrinogen, plasminogen, and plasminogen activators relatively free of plasmin inhibitors. Because the isoelectric point of vWF has been reported to be 5.7-5.9 (25), we expected it to be present in the precipitate also. Indeed, a $60-70 \%$ yield of vWF, with a multimer pattern indistinguishable from that in the starting plasma, was obtained in the euglobulin fraction. However, when the euglobulin fractions were clotted and allowed to lyse, no degradation of vWF multimers was observed. Hence, the sum of our efforts to demonstrate proteolysis of vWF in whole plasma, fibrin clots, or euglobulin fractions suggested that vWF is not a significant plasmin substrate in vivo.

\section{Acute effect of SK infusion on FVIII and $v W F$ in vivo}

Despite being unable to demonstrate plasmin degradation of vWF in whole plasma in vitro, we decided to examine the plasmas from patients receiving SK to determine whether multimer degradation occurred in vivo. A typical example of the acute effect of SK on the vWF multimers is shown in Fig. 5. Although we anticipated that, as plasmin was generated, the

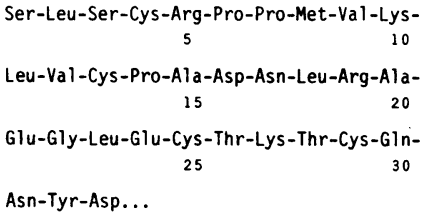
quence of P34.
Figure 4. Amino-terminal se-
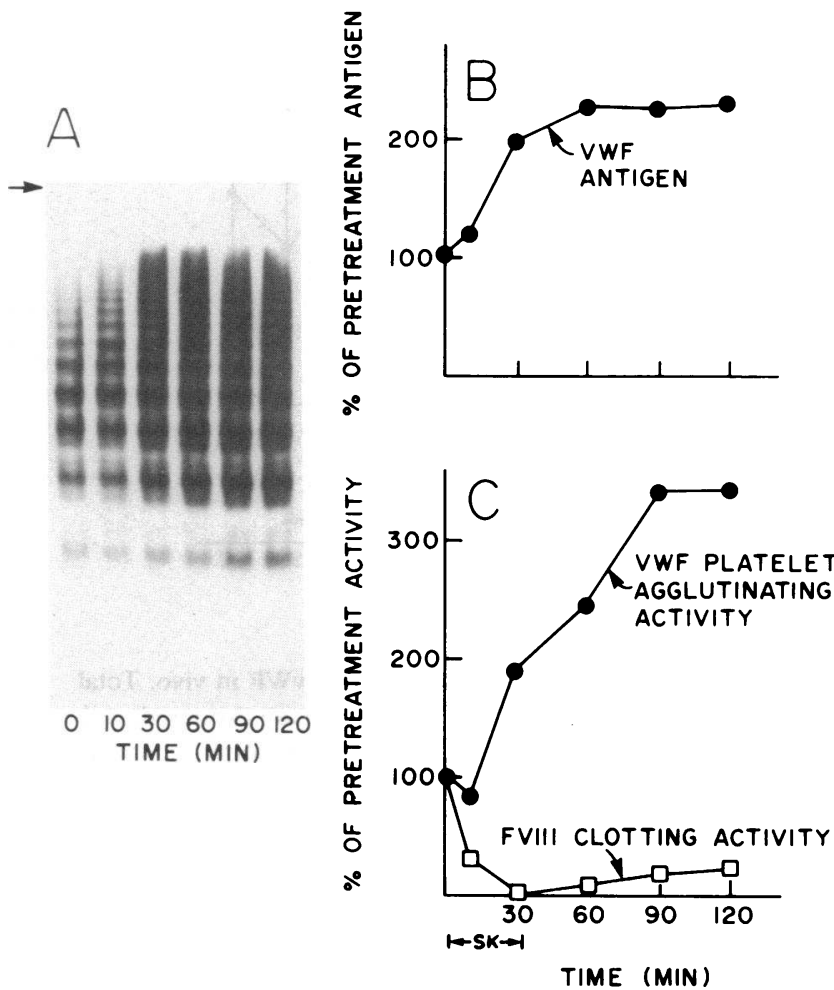

Figure 5. Typical acute response of FVIII/vWF after SK. This patient who had deep vein thrombosis received $250,000 \mathrm{U}$ of SK over 30 min. $(A)$ vWF multimer patterns analyzed by SDS-agarose gel electrophoresis and immunoblotting. $(B)$ Total vWF antigen quantitated from the radioimmunoblot. $(C)$ vWF platelet-agglutinating activity (ristocetin cofactor activity) and FVIII clotting activity (two-stage assay). Antigen and activity levels are each expressed as percent of pretreatment level.

multimers would be either unchanged or slightly degraded to smaller forms, we found instead that the level of vWF antigen (in all multimer sizes) was increased in 12 of 14 patients by $30 \mathrm{~min}$ after starting the SK and in all patients by $2 \mathrm{~h}$. The changes in vWF antigen, platelet-agglutinating activity, and FVIII clotting activity for these subjects are shown in Fig. 6. By $30 \mathrm{~min}$ after initiation of SK, total vWF antigen had increased to $150 \%$, and by $2 \mathrm{~h}$ the level was $193 \%$ of pretreatment level. This rapidly appearing vWF was apparently normal and functional because it was accompanied by an even greater increase in platelet-agglutinating activity, which reached $180 \%$ of base-line level by $30 \mathrm{~min}$, and remained elevated at 210 $260 \%$ for the next 90 min (Fig. 6). The plasma vWF levels in controls (who underwent cardiac catheterization or received a heparin loading dose) were unchanged. vWF antigen was also measured in small, medium, and large multimers, represented by multimers $1-4,5-8$, and 9 or larger, respectively. Although an increase in response to SK occurred in all three subpopulations of multimers (Fig. 7), the large forms showed the greatest increase: $290 \%$ of pretreatment level by $2 \mathrm{~h}$.

All but two patients had increased vWF antigen levels by the end of the single SK dose; however, during the first 5-20 min of the actual SK infusion, 8 of the 14 patients had transient decreases of 5-50\% in total vWF antigen and attendant losses in platelet-agglutinating activity. A typical example is shown in Fig. 8. The multimer patterns and quantitation of 


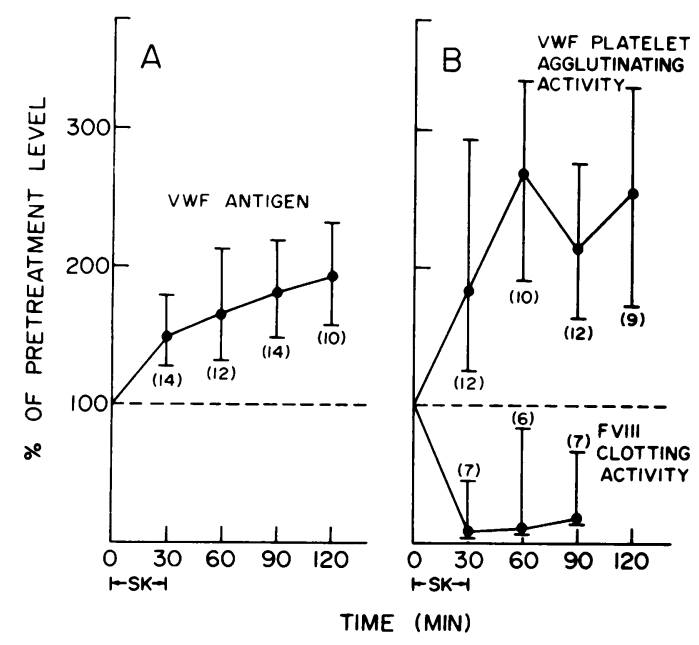

Figure 6. Effects of single SK dose on FVIII/vWF in vivo. Total vWF antigen $(A)$ and FVIII/vWF activity $(B)$ are expressed as the percentage of pretreatment levels. At each time point, the median value for $(n)$ subjects and the $95 \%$ confidence interval are shown.

antigen distribution among the small, medium, and large forms demonstrated decreases in all multimer populations, but the greatest decrease occurred in the large forms (data not shown). These findings are consistent with plasmin degradation plus clearance of some vWF from the circulation. The early decreases in VWF did not appear to be related to the dose of SK or to the patient's underlying disease, and were always followed by a rise in vWF antigen and platelet-agglutinating activity to above pretreatment levels, usually by $30 \mathrm{~min}$ and in all cases by $90 \mathrm{~min}$.

FVIII clotting activity could not be assayed by the onestage method, in that all patients had prolonged thrombin times after SK, presumably due to FDP. In the one-stage FVIII assay, the test plasma (diluted 1:5 to $1: 40$ ) was mixed with an equal volume of hemophilic plasma and Thrombofax before recalcification. When thrombin was substituted for calcium in this assay, mixtures containing post-SK plasma had prolonged clotting times compared to those with normal plasma or the patient's pretreatment plasma, suggesting that
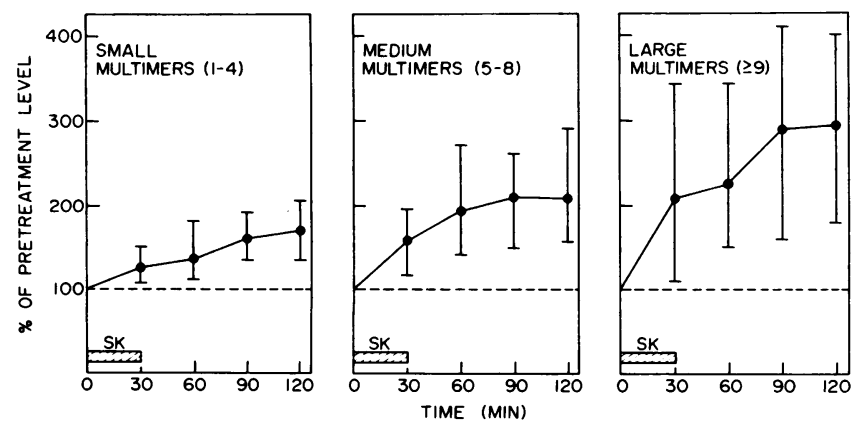

Figure 7. Differential effect of SK on small, medium, and large vWF multimers. The vWF antigen in multimers 1-4 (small), 5-8 (medium), and $\geq 9$ (large) was quantitated from radioimmunoblots. Results are expressed as the percentage of the pretreatment vWF antigen level in the appropriate multimer subgroup. Medians and their 95\% confidence intervals are shown; $(n)$ values are the same as those in Fig. $6 \mathrm{~A}$.

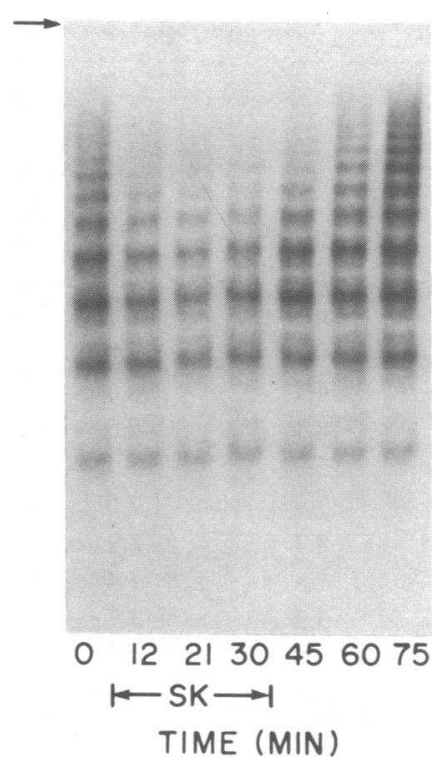

FDP interference with fibrin polymerization was obscuring FVIII measurement. In the two-stage FVIII assay, patient plasma (diluted 1:32 to 1:256) was further diluted 1:5 in the first stage incubation mixture and then 1:5 in the second stage. The FDP present in patient plasmas at these dilutions did not interfere with fibrin polymerization as demonstrated by normal clotting times when the two-stage assay mixture was clotted with thrombin instead of calcium. Hence, FVIII activity could be assessed by the two-stage assay even during SK treatment. Indeed, $\mathbf{3 0}$ min after starting the SK dose, FVIII procoagulant activity was $8 \%$ of its pretreatment level (Fig. 6), which coincided with the rapid increases in vWF antigen level and platelet-agglutinating activity. $1 \mathrm{~h}$ later, median FVIII activity was still only $18 \%$ of the pre-SK level.

\section{Effect of prolonged SK infusion on plasma $v W F$.}

Five patients in our study received SK for $>12 \mathrm{~h}$. In all of these patients, the initial rise in vWF antigen was sustained, but in most, the platelet-agglutinating activity declined. These changes were clearly demonstrated when the ratio of vWF platelet-aggregating activity to antigen was plotted for each patient over the course of therapy (Fig. $9 \mathrm{~A}$ ). There was an initial increase in the activity-to-antigen ratio (specific activity) after the SK loading dose, followed by a decline toward pretreatment level in every patient during continued SK infusion. Multimer analysis of plasmas from these patients allowed an explanation for this loss of activity: a shift in the multimer distribution occurred over time in some patients, with a progressive loss of the largest multimers and a gradual increase in smaller forms (Fig. 9, $B$ and $C$ ). Although the sample size is small, these observations suggest that plasmin degradation of vWF may occur during extended fibrinolytic therapy.

\section{Discussion}

Limited proteolysis is a common mechanism by which circulating proteins are activated and degraded. In vitro, FVIII is activated by thrombin (26) and inactivated by plasmin $(6,7$, 9) and protein $C(27)$. It is presumed that these proteinases modulate the rate of fibrin formation in vivo by changing 

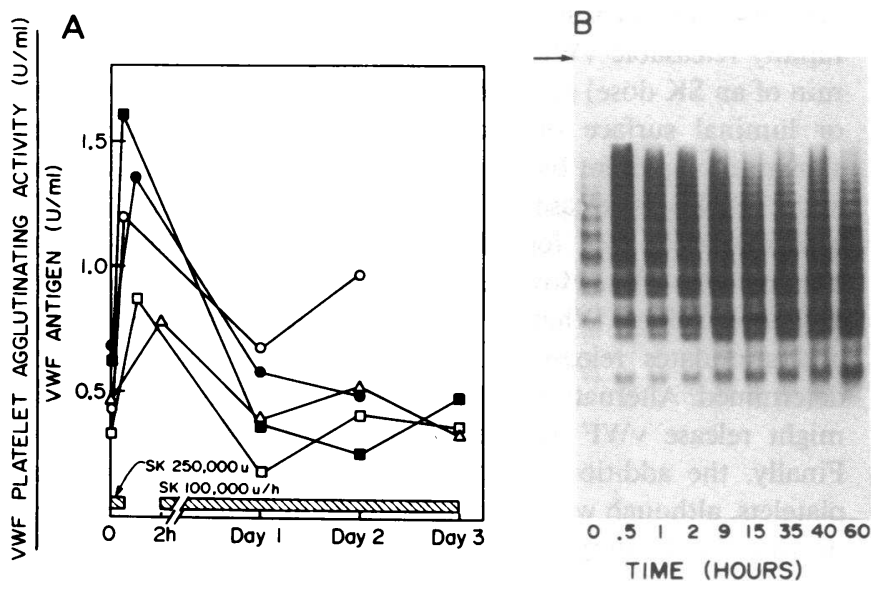

Figure 9. Effect of prolonged SK infusion on plasma vWF. $(A)$ The ratio of vWF platelet-agglutinating activity to $\mathrm{VWF}$ antigen in five patients treated for pulmonary embolus or deep vein thrombosis. The maximum ratio for each patient during the first $2 \mathrm{~h}$ after the loading dose is shown, along with values obtained on days 1,2 , and 3 of the SK infusion. $(B)$ vWF multimer analysis in one patient treated with

FVIII activity. vWF is not a known substrate for any proteinase in vivo and the regulation of its synthesis, release, size, activity, and clearance is not well understood. The present study addresses the effect of plasmin on vWF multimer structure in vitro and the question of whether plasmin significantly affects FVIII/vWF in vivo.

Plasmin degradation of $v W F$ in vitro: structural implications. Gel analyses of purified plasmin-degraded vWF (Figs. 1, $A$ and $C$ and 2), together with the electron microscopic appearance of the vWF protomer (Fowler, W. E., L. J. Fretto, K. K. Hamilton, H. P. Erickson, and P. A. McKee, manuscript submitted for publication), suggest a model for the plasmin

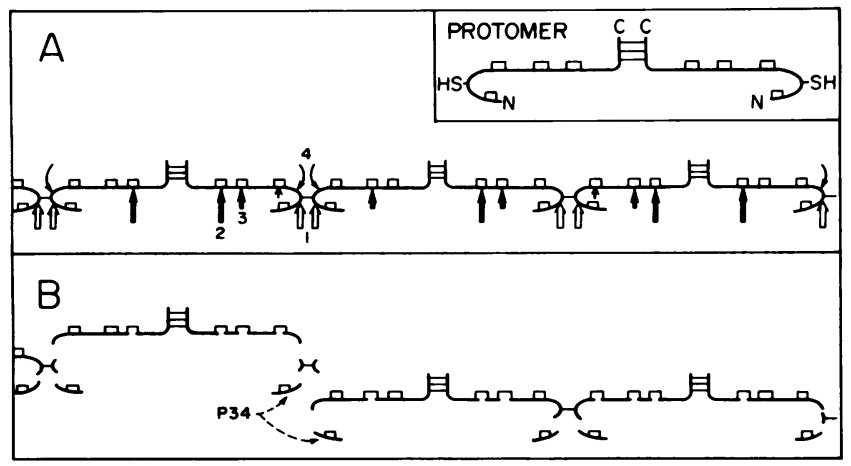

Figure 10. Model for plasmin degradation of vWF. The vWF protomer $(A$; inset $)$ is a dimer of 240,000 -mol wt subunits. Probable carboxy- and amino-termini are designated $C$ and $N$, respectively. Thin lines bridging the subunit(s) represent disulfide bonds. Although the dimer is relatively resistant to reducing agents, the number of intersubunit disulfide bonds is unknown. A portion of a vWF multimer is shown in $A$; large arrows represent approximate sites of preferred plasmin cleavages, one of which (arrow 1) releases a 34,000-mol wt amino-terminal fragment (P34). Others (arrows 2 and 3) occur within disulfide loops and generate 134,000 - and 114,000mol wt fragments. Minor cleavages are shown with thin arrows. At least one of these (arrow 4) cleaves the multimer chain very near the interprotomer disulfide bond(s). Typical products of plasmin digestion $(B)$ consist of small "multimers" and the P34 peptides.

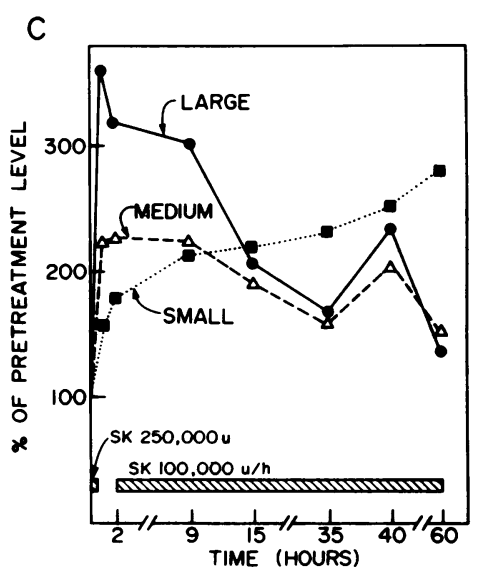

SK for $60 \mathrm{~h}$. (C) vWF antigen in small, medium, and large multimers (as defined in Fig. 7) quantitated from the immunoblot shown in $B$. The initial increase in all multimer sizes, followed by a gradual loss of large forms, and accumulation of small multimers with time (as seen in $B$ ) is confirmed.

degradation of vWF (Fig. 10). The vWF protomer (inset, Fig. $10 \mathrm{~A}$ ) is comprised of two identical subunits linked near their C-termini by disulfide bond(s) that are relatively resistant to reduction (2). The protomers are then linked into filamentous polymers (Fig. $10 \mathrm{~A}$ ) by disulfide bond(s) which are much more vulnerable to reducing agents. One rapid plasmin cleavage (arrow 1, Fig. $10 \mathrm{~A}$ ) releases the 34,000-mol wt fragment (P34), explaining the slight increase in electrophoretic mobility of the multimers (seen in Fig. $1 \mathrm{~A}$ as early as $5 \mathrm{~min}$ after the addition of plasmin). The amino-terminal sequence of P34 (Fig. 4) matches the 15 residues recently reported for the aminoterminal sequence of the intact vWF subunit (28). Most of the other plasmin cleavages must occur within disulfide loops, because the fragments that are generated remain disulfidelinked into multimers. Two large fragments $(134,000$ and $114,000 \mathrm{~mol} w \mathrm{w}$ ) are produced early, and preliminary Staphylococcus aureus V8 digest peptide patterns by SDS-PAGE suggest that these overlap each other and the $168,000-\mathrm{mol} w t$ fragment. In addition, V8 peptides of P34 are also present in the pattern of $V 8$ peptides from the 168,000 -mol wt fragment, but not in the V8 peptide patterns from the 134,000- and 114,000 -mol wt fragments. Hence, the 34,000- and 134,000mol wt fragments may compose the $168,000-\mathrm{mol}$ wt piece. Of note, in all previous studies of plasmin-degraded vWF, two large, rather stable fragments have been observed in terminal digests, although their molecular weight estimates have been quite variable: 103,000 and $88,000 \mathrm{~mol} \mathrm{wt}(9), 118,000$ and $98,000 \mathrm{~mol} w t(6)$, and 155,000 and $120,000 \mathrm{~mol} \mathrm{wt} \mathrm{(7).}$

Plasmin only slowly degrades the 134,000 - and 114,000 mol wt fragments. Most of these minor or slower cleavages (thin arrows, Fig. $10 \mathrm{~A}$ ) occur within disulfide loops, generating smaller fragments $(21,000 \mathrm{~mol} \mathrm{wt}$ and $43-78,000 \mathrm{~mol} \mathrm{wt})$ that remain bound within the multimers. However, at least one of these slower cleavages does not lie within a disulfide loop (arrow 4, Fig. $10 \mathrm{~A}$ ) and produces shorter vWF polymers which are still multiples of a nearly normal-sized, though fragmented, protomer (Fig. $10 \mathrm{~B}$ ). This cleavage site must therefore be very close to the interprotomer disulfide bond(s). With trypsin (Fig. $1 D$ ) the 134,000- and 114,000-mol wt 
fragments are generated early and then rapidly digested; their disappearance after $5 \mathrm{~h}$ (Fig. $1 D$ ) corresponds to the disappearance of multimers (Fig. $1 B$ ). Further cleavage of the 134,000 - and 114,000 -mol wt fragments by trypsin or plasmin parallels proteolysis of the large multimers, suggesting that the cleavage that produces shorter multimers probably occurs within these two fragments. The incomplete degradation of the multimers (and specifically their 134,000- and 114,000mol wt fragments), despite repeat additions of plasmin, suggests that as degradation proceeds, vWF becomes progressively resistant to further proteolysis. Thus major changes in vWF structure may occur during digestion which causes initially available cleavage sites to become poorly accessible to plasmin, whereas trypsin, with its smaller size and broader specificity, completes its digestion.

Plasmin effects on FVIII in vivo. In vitro degradation of FVIII/vWF by plasmin does not imply that either of these proteins is a significant physiologic plasmin substrate. We did find, however, that addition of SK to plasma destroyed FVIII activity, suggesting that plasmin inactivates FVIII even in the presence of fibrinogen. Although textbooks and reviews of thrombolytic therapy $(29,30)$ often state that plasmin cleavage of FVIII, in addition to fibrin and fibrinogen, contributes to the "lytic state," few patient studies have been performed (3133), and the effect of FDP on the FVIII assays used in those studies was not addressed. Lombardi et al. (33) examined FVIII activity in patients receiving urokinase therapy for deep vein thrombosis and found an insignificant change by the onestage assay and a significant decrease (from 163 down to 96 $\mathrm{U} / \mathrm{dl}$ ) by the two-stage method in eight patients after $6 \mathrm{~h}$ of urokinase. In contrast, we could not use the one-stage FVIII assay for our patients receiving SK in that FDP levels were sufficient to inhibit fibrin polymerization. However, the standard two-stage assay (21) circumvented this problem, and we found that SK-induced fibrinolysis was accompanied by decreases in FVIII procoagulant activity to $10-20 \%$ of its pre-SK level (Fig. 6).

$S K$ effects on $v W F$ in vivo: stimulation of $v W F$ release and modulation of activity. In contrast to FVIII, we were unable to detect plasmin degradation of VWF in whole plasma in vitro, and therefore suspected that vWF may not be an important plasmin substrate in vivo. Nevertheless, we reasoned that if plasmin-degraded vWF multimers could be generated in vivo, we should find them in the plasmas of patients receiving SK. Surprisingly, however, rather than degradation, a twofold median increase in vWF antigen and plateletagglutinating activity occurred after a single dose of SK (Fig. 6). Lombardi et al. (33) did not find increased vWF antigen in their urokinase-treated patients by immunoradiometric assay, but they did find an increase by rocket immunoelectrophoresis that was attributed to proteolysis of vWF. They observed a new fast-moving peak (also seen in patients with disseminated intravascular coagulation) by radio-crossed immunoelectrophoresis when eight to nine times the usual plasma load was applied and suggested this to be a minor cleavage fragment of vWF. After a 30-min SK infusion, we documented an increase in vWF antigen (Fig. 6), which was comprised of multimers of all sizes (Fig. 7) and cannot be explained by proteolytic modification of already circulating vWF. It appears that SK, presumably via either the SK-plasminogen activator complex, plasmin, or plasmin degradation products of other proteins (e.g., FDP), stimulates rapid release of vWF from endothelial cells, platelets, or some as yet unrecognized storage pool. This rapidly releasable vWF (often appearing within the first 5-10 min of an SK dose) probably comes from the secretory vesicles or luminal surface of endothelial cells, where it has been shown to be present by electron microscopy $(34,35)$. Cultured endothelial cells release vWF in response to thrombin $(36,37)$, a serine proteinase for which these cells have high-affinity binding sites (38). However, thrombin does not cleave vWF as plasmin does. Whether plasmin binds to endothelial cells and stimulates release of intracellular vWF remains to be determined. Alternatively, by very selective proteolysis, plasmin might release vWF attached to the endothelial cell surface. Finally, the additional vWF might have been derived from platelets, although we were unable to demonstrate vWF release in vitro by treating platelet-rich plasma with SK. That the newly released vWF multimers were predominantly large explains the greater increase in platelet-agglutinating activity than in total vWF antigen and also provides some insight into the multimer distribution within the acutely releasable vWF storage pool.

Many stimuli such as exercise, catecholamines, and vasopressin increase both FVIII/vWF and plasminogen activator activity in plasma (39-41). However, neither plasminogen activators nor plasmin has ever been suggested to cause vWF release. There is an important difference between the FVIII/ vWF response to such stimuli as exercise or vasopressin and its response to SK: after SK, only vWF increases while FVIII activity rapidly falls. To our knowledge, there are no other physiologic or pharmacologic stimuli that cause simultaneous, profound increases in vWF activity and decreases in FVIII activity.

Although the major effect of SK was a dramatic increase in $\mathrm{vWF}$ antigen and platelet-agglutinating activity, we did observe probable plasmin degradation of $\mathrm{vWF}$, albeit very transiently, during the first 5-20 min of SK in about half our patients (Fig. 8). It is likely that some degradation and clearance of vWF occurred in all of these patients and the release of new VWF is superimposed on these processes. Which effect predominates early after SK must depend on the rate and magnitude of each response in a given patient. Later (after 30-60 $\mathrm{min}$ ), the release of new vWF consistently outweighed any degradation or clearance, such that $\mathrm{vWF}$ levels remained elevated. Perhaps some form of feedback regulation of plasma FVIII/vWF exists, wherein a fall in FVIII or vWF concentration or the presence of circulating FVIII/vWF degradation products triggers release of one or both proteins. During SK therapy that extended for 1-3 days, a relative loss of platelet-agglutinating activity occurred in most patients, and a downward shift in the size of the multimer population (consistent with plasmin degradation) occurred in some. Loss of larger multimers, however, is not definitive evidence of plasmin cleavage, as such a shift might also occur if endothelial cells, under continuous stimulation to release vWF, were unable to assemble the very largest multimers, or if large forms were preferentially consumed.

In our patients who received prolonged SK therapy, the high levels of vWF antigen relative to vWF platelet-agglutinating and FVIII clotting activities are similar to those reported in a group of 100 patients with acute respiratory failure (42). Patients with mild lung injury showed an increase in slowmoving (large) vWF by crossed immunoelectrophoresis (similar to the immediate response to SK we observed), whereas those 
with severe lung injury showed an absence of slow forms and fourfold increases in rapidly migrating forms (similar to the changes observed in some patients after prolonged SK). The authors concluded that plasmin was not responsible for high vWF antigen levels because treatment of vWF with plasmin in vitro did not increase antigenicity. However, we did observe a consistent increase in vWF antigen in vivo after SK, suggesting that plasmin may contribute to the FVIII/vWF abnormalities reported in acute respiratory failure by stimulating $v W F$ release and then causing its partial degradation.

Some of our findings may have important physiologic and therapeutic implications. First, patients treated with SK can have very high levels of vWF which might predispose to excessive platelet adhesion in areas of ulcerated endothelium that are reexposed after thrombolysis. Such platelet masses may contribute to the early reocclusions that can occur when single doses of SK or short SK infusions are used to lyse intraarterial thrombi, as in the treatment of acute myocardial infarction (30). Secondly, plasminogen activators and/or plasmin may be very important in stimulating release and modulating the size and activity of vWF. The physiologic interactions of plasminogen activators and plasmin with the endothelium and the circulating FVIII/vWF complex deserve further investigation.

\section{Acknowledgments}

We thank Dr. Rodney Hewick, Genetics Institute, Cambridge, MA, for performing the partial amino acid sequence of our P34 vWF peptide.

This work was supported in part by National Heart, Lung, and Blood Institute grants HL-15615 and HL-07101 and by a North Carolina Heart Association Grant-in-Aid Award 1983-1984-A-17.

\section{References}

1. Turitto, V. T., H. J. Weiss, and H. R. Baumgartner. 1984. Platelet interaction with rabbit subendothelium in von Willebrand's disease: altered thrombus formation distinct from defective platelet adhesion. J. Clin. Invest. 74:1730-1741.

2. Counts, R. B., S. L. Pashell, and S. K. Elgee. 1978. Disulfide bonds and the quarternary structure of factor VIII/von Willebrand factor. J. Clin. Invest. 62:702-709.

3. Perret, B. A., M. Furlan, and E. A. Beck. 1979. Studies on factor VIII-related protein. II. Estimation of molecular size differences between factor VIII oligomers. Biochim. Biophys. Acta. 578:164-174.

4. Hoyer, L. W., and J. R. Shainoff. 1980. Factor VIII-related protein circulates in normal human plasma as high molecular weight multimers. Blood. 55:1056-1059.

5. Ohmori, K., L. J. Fretto, R. L. Harrison, M. E. P. Switzer, H. P. Erickson, and P. A. McKee. 1982. Electron microscopy of human factor VIII/von Willebrand glycoprotein: effect of reducing reagents on structure and function. J. Cell. Biol. 95:632-640.

6. Atichartakarn, V., V. J. Marder, E. P. Kirby, and A. Z. Budzynski. 1978. Effects of enzymatic degradation on the subunit composition and biologic properties of human factor VIII. Blood. 51: 281-297.

7. Guisasola, J. A., C. G. Cockburn, and R. M. Hardisty. 1978. Plasmin digestion of factor VIII: characterization of the breakdown products with respect to antigenicity and von Willebrand activity. Thromb. Haemostasis. 40:302-315.

8. Switzer, M. E. P., and P. A. McKee. 1979. Immunologic studies of native and modified human factor VIII/von Willebrand factor. Blood. 54:310-321.

9. Andersen, J. C., M. E. P. Switzer, and P. A. McKee. 1980.
Support of ristocetin-induced platelet aggregation by procoagulantinactive and plasmin-cleaved forms of human factor VIII/von Willebrand factor. Blood. 55:101-108.

10. Lian, E. C., R. L. Nunez, and D. R. Harkness. 1976. In vivo and in vitro effects of thrombin and plasmin on human factor VIII (AHF). Am. J. Hematol. 1:481-491.

11. Newman, J. A., A. J. Johnson, M. H. Karpatkin, and S. Puszkin. 1971. Methods for the purification of clinically effective intermediate- and high-purity factor-VIII concentrates. Br. J. Haematol. 21:1-20.

12. Sodetz, J. M., S. V. Pizzo, and P. A. McKee. 1977. Relationship of sialic acid to function and in vitro survival of human factor VIII/ von Willebrand factor protein. J. Biol. Chem. 252:5538-5546.

13. Lowry, O. H., N. J. Rosebrough, A. L. Farr, and R. J. Randall. 1951. Protein measurement with the Folin phenol reagent. J. Biol. Chem. 193:265-275.

14. Deutsch, D. G., and E. T. Mertz. 1970. Plasminogen purification from human plasma by affinity chromatography. Science (Wash. DC). 170:1095-1096.

15. Switzer, M. E., and P. A. McKee. 1976. Studies on human antihemophilic factor: evidence for a covalently linked subunit structure. J. Clin. Invest. 57:925-937.

16. Fretto, L. J., E. W. Ferguson, H. W. Steinman, and P. A. McKee. 1978. Localization of the $\alpha$-chain cross-link acceptor sites of human fibrin. J. Biol. Chem. 253:2184-2195.

17. Hewick, R. M., M. W. Hunkapiller, L. E. Hood, and W. J. Dreyer. 1981. A gas-liquid solid phase peptide and protein sequenator. J. Biol. Chem. 256:7990-7997.

18. Head, D. R., R. P. Bowman, D. J. Manner, and A. D. Brossoit. 1979. An improved assay for von Willebrand factor. Am. J. Clin. Pathol. 72:991-995.

19. Langdell, R. D., R. H. Wagner, and K. M. Brinkhous. 1953. Effect of antihemophilic factor on one-stage clotting tests. J. Lab. Clin. Med. 41:637-647.

20. Kopitsky, R. G., M. E. P. Switzer, and P. A. McKee. 1982. Thrombin potentiation of factor VIII procoagulant activity: assessment by the two-stage assay. Thromb. Haemostasis. 47:145-149.

21. Biggs, R. 1976. Human Blood Coagulation, Haemostasis and Thrombosis. 2nd Ed. Blackwell Scientific Publications, Oxford. 684688; 721-722.

22. Laemmli, U. K., and M. Favre. 1973. Maturation of the head of the bacteriophage T4. I. DNA packaging events. J. Mol. Biol. 80: 575-599.

23. Merril, C. R., D. Goldman, S. A. Sedman, and M. H. Ebert. 1981. Ultrasensitive stain for proteins in polyacrylamide gels shows regional variation in cerebrospinal fluid proteins. Science (Wash. DC). 211:1437-1438.

24. Hollander, M., and D. Wolfe. 1973. Nonparametric Statistical Methods. John Wiley \& Sons, New York. 26-38.

25. Fulcher, C. A., Z. M. Ruggeri, and T. S. Zimmerman. 1983. Isoelectric focusing of human von Willebrand factor in urea-agarose gels. Blood. 61:304-310.

26. Switzer, M. E. P., and P. A. McKee. 1980. Reactions of thrombin with human factor VIII/von Willebrand factor. J. Biol. Chem. 255:10606-10611.

27. Fulcher, C. A., J. E. Gardner, J. H. Griffin, and T. S. Zimmerman. 1984. Proteolytic inactivation of human factor VIII procoagulant protein by activated human protein $\mathrm{C}$ and its analogy with factor V. Blood. 63:486-489.

28. Hessel, B., H. Jörnvall, L. Thorell, S. Söderman, U. Larsson, N. Egberg, B. Blombäck, and A. Holmgren. 1984. Structure-function relationships of human factor VIII complex studied by thioredoxin dependent disulfide reduction. Thromb. Res. 35:637-651.

29. Chesterman, C. N. 1981. Thrombolytic therapy. In Haemostasis and Thrombosis. A. L. Bloom, and D. P. Thomas, editors. Churchill Livingstone, Edinburgh. 693-711. 
30. Laffel, G. L., and E. Braunwald. 1984. Thrombolytic therapy. A new strategy for the treatment of acute myocardial infarction. $N$. Engl. J. Med. 311:710-717; 770-776.

31. McNicol, G. P., S. B. Gale, and A. S. Douglas. 1963. In-vitro and in-vivo studies of a preparation of urokinase. Br. Med. J. 1:909915.

32. Schöffel, G., R. Zimmerman, J. Harenburg, and H. Mörl 1982. Blood coagulation changes during effective thrombolysis using urokinase and heparin. Thromb. Res. 25:11-21.

33. Lombardi, R., P. M. Mannucci, M. J. Seghatchian, V. V. Garcia, and R. Coppola. 1981. Alterations of factor VIII von Willebrand factor in clinical conditions associated with an increase in its plasma concentration. Br. J. Haematol. 49:61-68.

34. Jeanneau, C., and Y. Sultan. 1982. Localization of factor VIII/ von Willebrand factor antigen by immunoelectron microscopy in human endothelial cells using Fab fragments coupled to peroxidase. $J$. Histochem. Cytochem. 30:1091-1096.

35. Rand, J. H., I. I. Sussman, R. E. Gordon, S. V. Chu, and V. Solomon. 1980. Localization of factor-VIII-related antigen in human vascular subendothelium. Blood. 55:752-756.

36. Levine, J. D., J. M. Harlan, L. A. Harker, M. L. Joseph, and R. B. Counts. 1982. Thrombin-mediated release of factor VIII antigen from human umbilical vein endothelial cells in culture. Blood. 60: 531-534.
37. de Groot, P. G., M. D. Gonsalves, C. Loesberg, M. F. van Buul-Wortelboer, W. G. van Aken, and J. A. van Mourik. 1984. Thrombin-induced release of von Willebrand factor from endothelial cells is mediated by phospholipid methylation. J. Biol. Chem. 259: 13329-13333.

38. Aubrey, B. J., J. C. Hoak, and W. G. Owen. 1979. Binding of human thrombin to cultured human endothelial cells. J. Biol. Chem. 254:4092-4095.

39. Ruggeri, Z. M., P. M. Mannucci, R. Lombardi, A. B. Federici, and T. S. Zimmerman. 1982. Multimeric composition of factor VIII/ von Willebrand factor following administration of DDAVP: implications for pathophysiology and therapy of von Willebrand's disease subtypes. Blood. 59:1272-1278.

40. Nilsson, I. M., H. Vilhardt, L. Holmberg, and B. Åstedt. 1982. Association between factor VIII related antigen and plasminogen activator. Acta Med. Scand. 211:105-112.

41. Mannucci, P. M., M. Aberg, I. M. Nilsson, and B. Robertson. 1975. Mechanism of plasminogen activator and factor VIII increase after vasoactive drugs. Br. J. Haematol. 30:81-93.

42. Carvalho, A. C. A., S. M. Bellman, V. J. Saullo, D. Quinn, and W. M. Zapol. 1982. Altered factor VIII in acute respiratory failure. N. Engl. J. Med. 307:1113-1119. 